\title{
Development and Application of Three-Dimensional Virtual Simulation Training System of Coal Mine
}

\author{
Jie Chen ${ }^{1,2}$, Yi He ${ }^{*}, 1$, Deyi Jiang ${ }^{1}$ and Xiao Guo ${ }^{3}$ \\ ${ }^{1}$ State Key Laboratory for the Coal Mine Disaster Dynamics and Controls, Chongqing University, Chongqing 400044, \\ China \\ ${ }^{2}$ Institute of Rock and Soil Mechanics, The Chinese Academy of Science, Wuhan, Hubei 430071, China \\ ${ }^{3}$ Chongqing perfect Technology Co. Ltd, Chongqing 400000, China
}

\begin{abstract}
This paper studies the application of virtual simulation technology in coal mine, and proposes threedimensional virtual simulation training system for coal mines. This system mainly includes "finger oral" and reduction of accident cases. This paper describes the characteristics and development process of three-dimensional virtual simulation training system which can achieve three-dimensional visual effects combining three-dimensional stereoscopic projection technology. The field application shows that three-dimensional virtual simulation training system developed in this paper has achieved better training affects, greatly improved the quality of coal mine safety training and enhanced the enthusiasm of employee, and promoted the safe production of coal mine. Few patents associated with virtual simulation are discussed in this paper.
\end{abstract}

Keywords: Accident cases, three-dimensional virtual simulation, training system.

\section{INTRODUCTION}

With the rapid development of information technology, China's coal enterprises are developing forward in the direction of large-scale and high efficiency field, but China's coal mine casualties have accounted for about $70 \%$ of the world casualtiy figures, according to statistics, approximately $97 \%$ of the mine disasters are caused by human error [1]. Therefore, to effectively improve safety awareness, education and skills in production safety for staff in coal mines is extremely important. Most traditional ways of coal mine safety training and education in China are production safety theory, case study as well as writing materials, lack of comprehensive information of field environment leads to the effect of unideal safety training.

There is an urgent demand for innovative safety training methods of relevance and applicability [2, 3]. At present, the virtual simulation technology has been gradually applied to the management and decision-making process of the coal industry. It can not only simulate the normal working environment in a coal mine, but can also simulate coal mine emergency rescue and disaster relief environment and analyze typical coal mine accidents, so that mine workers can experience the virtual environment of coal mine production, grasp the causes of the accident and learn to deal with the production process safety issues [4-6]. Therefore, this paper develops the three-dimensional virtual simulation training system, describes post operation and key points

*Address correspondence to this author at the State Key Laboratory for the Coal Mine Disaster Dynamics and Controls, Chongqing University, Chongqing 400044, China; Tel: 86-10-84671739; Fax: 86-10-84671739; E-mail: yoyaheyi@qq.com through animation, voice, text prompts, achieves the goal of the coal mine job types by "fingers oral" and accident cases restore function. The system is compared with traditional coal mine safety training and education. It reduces training costs, and improves worker safety training. Presently, the system has been applied to a mine in Shanxi Yangquan Coal Group and achieved better result.

\section{FEW PATENTS ON VIRTUAL SIMULATION}

There are many researchers studying the virtual simulation. In a test, which uses simulators corresponding to each component to be simulated, communication between components can be easily observed and therefore protecting information that should be concealed is becoming difficult [7]. Patent US14047803, titled "System and method for the configuration of a clustered simulation network" [8] provides a system and method for configuring a clustered simulation network using virtualization. A simulator facilitates virtual welding activity of orbital weld joints [9]. Patent US13942965, titled "Automatic context management for web application with client side code execution" [10] the invention can record and replay context-full scripts that do not require manual customization and are capable of handling state information even for web applications that execute code on the client side. The robot simulator is provided with a display unit, an image-generating section (111a), a display control section (111b), and a simulation instruction section (111e) [11]. The present invention enables measuring the size of a displayed object with a measuring tape tool, which is generated when selected with a mouse, and changes the display according to the desired scale. In accordance with the size of the object that is 
inputted by a user, to allow arranging interior design items such as stickers, frames, and furniture in a virtual space in a variety of ways, thereby enabling accurate interior design in a real space and enhancing results [12]. Patent US14004938, titled "Sensing device and method used for virtual golf simulation apparatus" [13] discloses a sensing device and method used for a virtual golf simulation apparatus in which an image acquired by an inexpensive camera having a relatively low resolution and velocity is analyzed to relatively accurately extract information on physical properties. A work state virtual simulation system for an electric haulage shearer based on different geological conditions belongs to automatic control and system simulation of shearers. The work state virtual simulation system comprises of devices and a method. The devices consist of a shearer work condition remote monitoring platform, a database, a remote controller, a visual basic system, an industrial Ethernet, a local controller and a machine-mounted detection control system [14]. Coal mine accident simulating method and system based on multiintelligent agent [15] can be used for imitating coal mine accidents in various environments to provide abundant information for discovering the coal mine accident potential.

\section{THREE-DIMENSIONAL VIRTUAL SIMULATION TRAINING SYSTEM OF COAL MINE}

The 3D virtual simulation training system developed in this paper includes two functions: the coal mine job types by fingers and oral and the reconstruction of coal mine accident cases. The system structure is shown in Fig. (1). This system produced mainly refers to "coal mine production safety" and the method of "finger oral" and so on. Among them, the main points of manipulation and the method of "finger oral" show coal mine job types by fingers and oral through the form of 3D animation, mine accident cases including the accident of ventilation and control of gas, fire and coal dust, roof collapse, transport accidents and electrical and mechanical accidents, to educate and improve the safety awareness of workers through the virtual simulation of accident case.

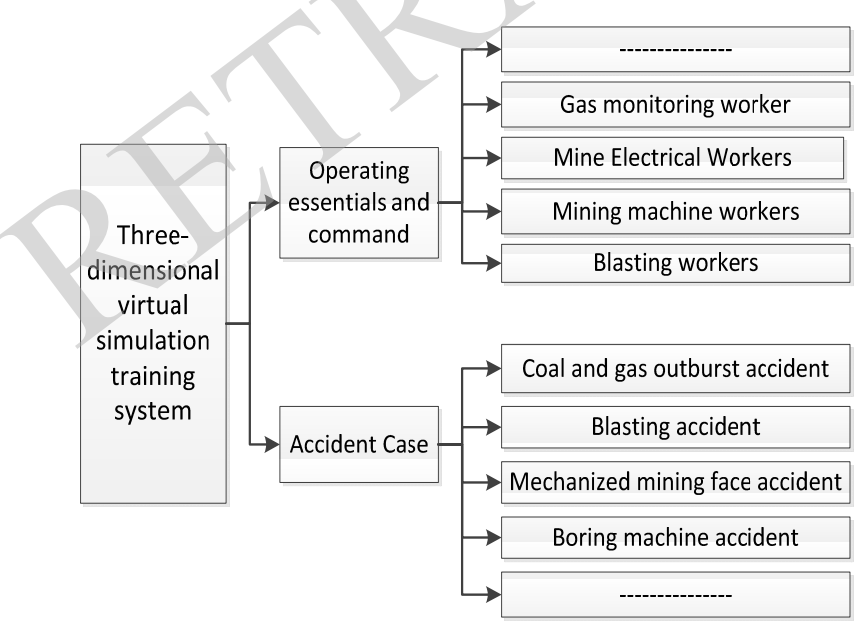

Fig. (1). The system structure.

The development process of this system are field investigation/the collection of accident reports and material $\rightarrow$ construction of scene models and characters by 3DMAX $\rightarrow$ drawing the map $\rightarrow$ the lighting modulation $\rightarrow$ modulation of animation and character $\rightarrow$ rendering sequence frame $\rightarrow$ dubbing, audio production and so on, it can be summarized as pre-production, the production process and post-synthesis, a detailed introduction follows.

\subsection{Pre-Production}

Firstly, the system does a field investigation, the analysis of the accident process and report and field scene and so on before production in order to enable developers to have a general understanding of the whole accident by the field investigation. The live scene is used for the developers to have a deeper understanding of the underground environment which can realistically simulate the underground environment, character and workflow.

\subsection{The Production Process}

After the completion of pre-production 3D modeling software $3 \mathrm{DMAX}$ is used to create animation process that mainly includes the production of the mine environment model, role model, texture, character animation, smoke, fire, water, explosion and other effects and the control and rendering of the camera and so on.

\subsubsection{The Mine Environment and Role Modeling}

The scenic environment of the 3D virtual simulation training system includes static roadway models, electromechanical equipment and dynamic personnel, mobile devices etc. This system adopts the geometric modeling method to build a static model of physical shape and size by using 3DMAX software, and then increase texture mapping, lights and shadows on the appearance of virtual model according to the corresponding material reference. For the entity model of complex and irregular shapes, we will trim the AutoCAD schematic of entity models, and then import it in 3DMAX, to construct the virtual model based on the contour of the entity model. Fig. (2a) depicts the virtual model of roadway. The virtual human modeling is based on geometric model and human body skeleton model. According to the analysis of the body's movement, modeling is based on non-uniform rational B-spline (NURBS) surface shape, combining with constructive solid geometry (CSG) to create the human body model [6]. As shown in Fig. (2b) for the characters of the virtual model.

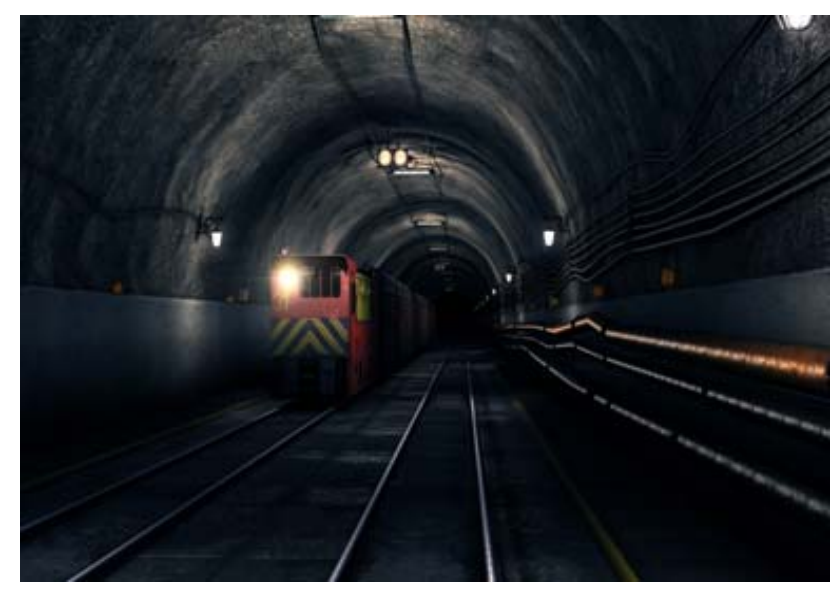

Fig. (2a). The virtual model of roadway. 


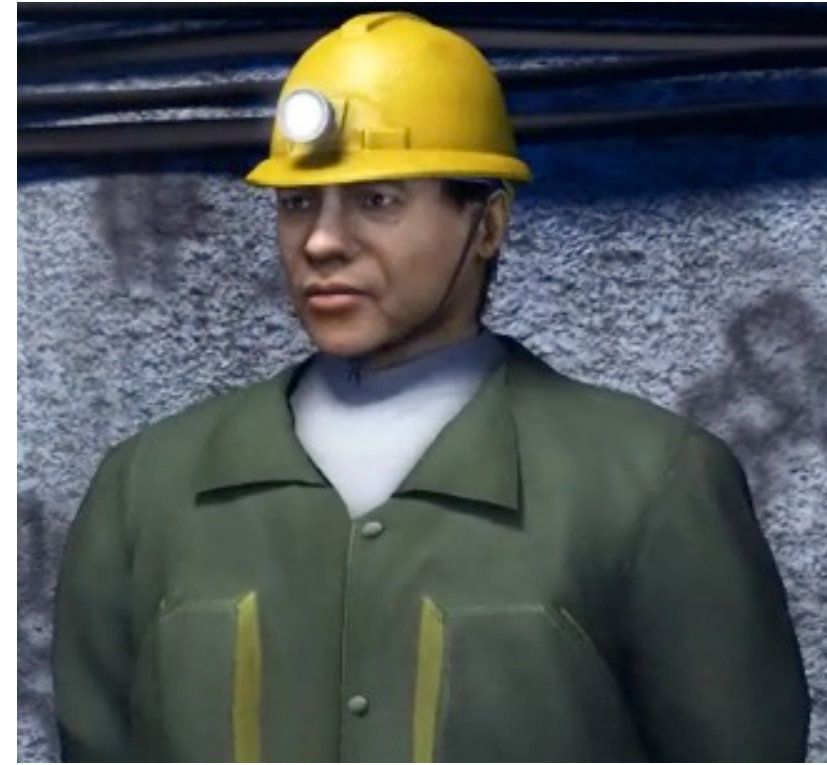

Fig. (2b). The characters of the virtual model.

\subsubsection{Texture Mapping}

Texture mapping builds a model of texture by mapping textures to the model appearance, to enhance the authenticity of model. The pictures of the desired object are modified and pasted on to the three-dimensional model by the Photoshop software, forming the texture and structure of the model appearance. The texture may not match UV position of three-dimensional entity model in this process, and by adopting the Unwrap UVW command of the modifier list to amend the texture and correctly map to the model in 3DMAX

\subsubsection{Lights}

Lights can enhance the quality of the scene in the virtual environment. So choosing the suitable lighting plays an important role and effectiveness of the three-dimensional virtual technologies. V-Ray rendering plug-in is used which has fast rendering speed and the effect of the picture is realistic under normal circumstances. V-Ray's surface and ambient light and the default point light are uitlised to render effects of light and shade for the scenic lighting.

\subsubsection{Character Animation}

The mine environment only reflects the inherent characteristics of the virtual scene; it cannot show the movement of the virtual object characteristics. Therefore, character animation is an important step to make the 3D virtual scene realistic. The virtual environment and virtual characters of the coal mine are completed by the appropriate action through key frame control and bones in 3DMAX and converted into an animated sequence of frames.

\subsubsection{The Camera Control}

Along with character movement, the ordinary threedimensional virtual simulation system requires a camera that follows the movement of the character. The threedimensional simulation training system developed in this paper requires a combination of 3D stereoscopic projection technology to display the three-dimensional visual effects, so two cameras are used.

\subsubsection{Rendering Frame for Animation Sequences}

Scene rendering is used to observe how realistic the virtual environment is. Factors such as the arrangement of lights, adjusting the light in time, coloring model of the scene, making the scene model, materials and lighting process images are considered.

\subsection{Post-Synthesis}

Using Combustion or Nuke to add specular highlights channel, Z channel, self-illumination channel and some special frames to form the final animation file. By integrating frames of animation sequence, dubbing, adding sound effects together through the Adobe Premiere Pro tool, and adding subtitles to the corresponding sequences and ultimately generating a complete simulation animation training systems.

\section{EXAMPLES OF APPLICATION SYSTEM}

$3 \mathrm{D}$ virtual simulation training system in the paper has been successfully úsed in Yangquan Coal Group in Shanxi province. It includes 16 types of post work accidents in the coal mine and 26 types of typical accident cases, such as, the accident of ventilation and control of gas, fire and coal dust, roof accident, transportation accident, mechanical and electrical injuries and so on. The following example will introduce the process in detail.

\subsection{Events Leading Upto the Accident}

The drilling team has finished drilling shot hole at $6 \mathrm{am}$, and prepared the charge. Charge man and blasting-technician went to the main roadway to charge and initiate blast. Dong took charge to withdraw and remove the miners from the site and placed a safety red line. Coal and gas outburst occurred after the explosion at $6: 40 \mathrm{am}$. The miners withdrew to a zone only $7 \mathrm{~m}$ away and then panicked at the sight and starting fleeing. This confusion and chaos resulted in the death of a miner.

Cause of accident:

1) The construction site of utilizes $3 \#$ outburst coal seam. Outburst prevention measures are imperfect and are direct cause of the accident.

2) Blasters illegal explosive initation resulted in coal and gas outburst, and the position where the miners' withdrew was inappropriate, the distance is not enough, which is the main reason that caused the accident.

3) The method to avoid disaster was improper. In the process of withdrawal the staff was tripped by storage of cement on the roadway. The victim inhaled high concentrations of gas and died of suffocation, and it is an important cause of the accident.

4) Managers at all levels lack the knowledge of outburst prevention and are not strict about the implementation 
of outburst prevention measures. It is one of the important reasons of the cause of the accident.

Precautionary measure:

1) Re-correction of procedural measures;

2) Retrain the technical management personnel and onsite management personnel;

3) Earnestly implement the responsibility system for production safety and strengthen field management;

4) Strengthen safety training and education to improve the safety awareness of all employees.

Firstly, build model for the virtual characters and the environment in this case, virtual work environment as shown in Fig. (3). Then perform animation for simulation aimed at mine workers' illegal behavior in this case, as shown in Fig. (4). At last, making animation to simulate the consequences arising out of the illegal operation, as shown in Fig. (5). It is showed that from the above diagram due to the illegal explosion by workers led to coal and gas outburst which caused the death of a co-worker. The system developed in this paper reconstructs the whole process of the accident, and displays the cause of the accident and prevention measures in the animation video, demonstrates the serious consequences of the accident and plays a warning.

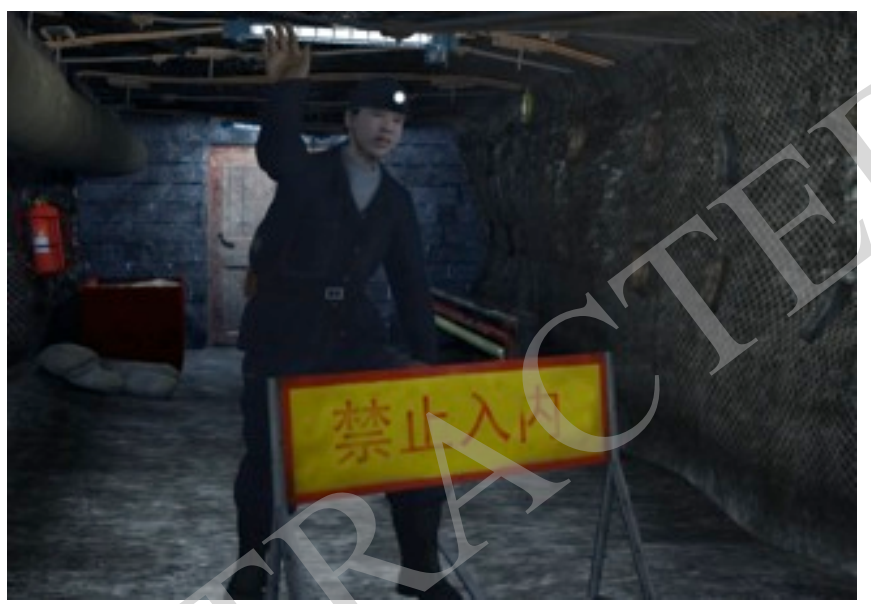

Fig. (3). Virtual work environment.

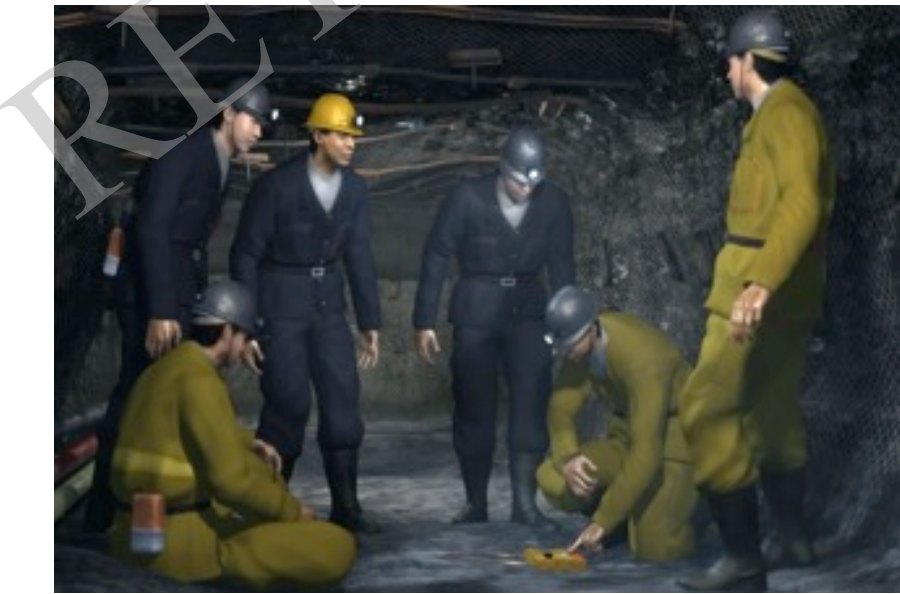

Fig. (4). Illegal behaviors.
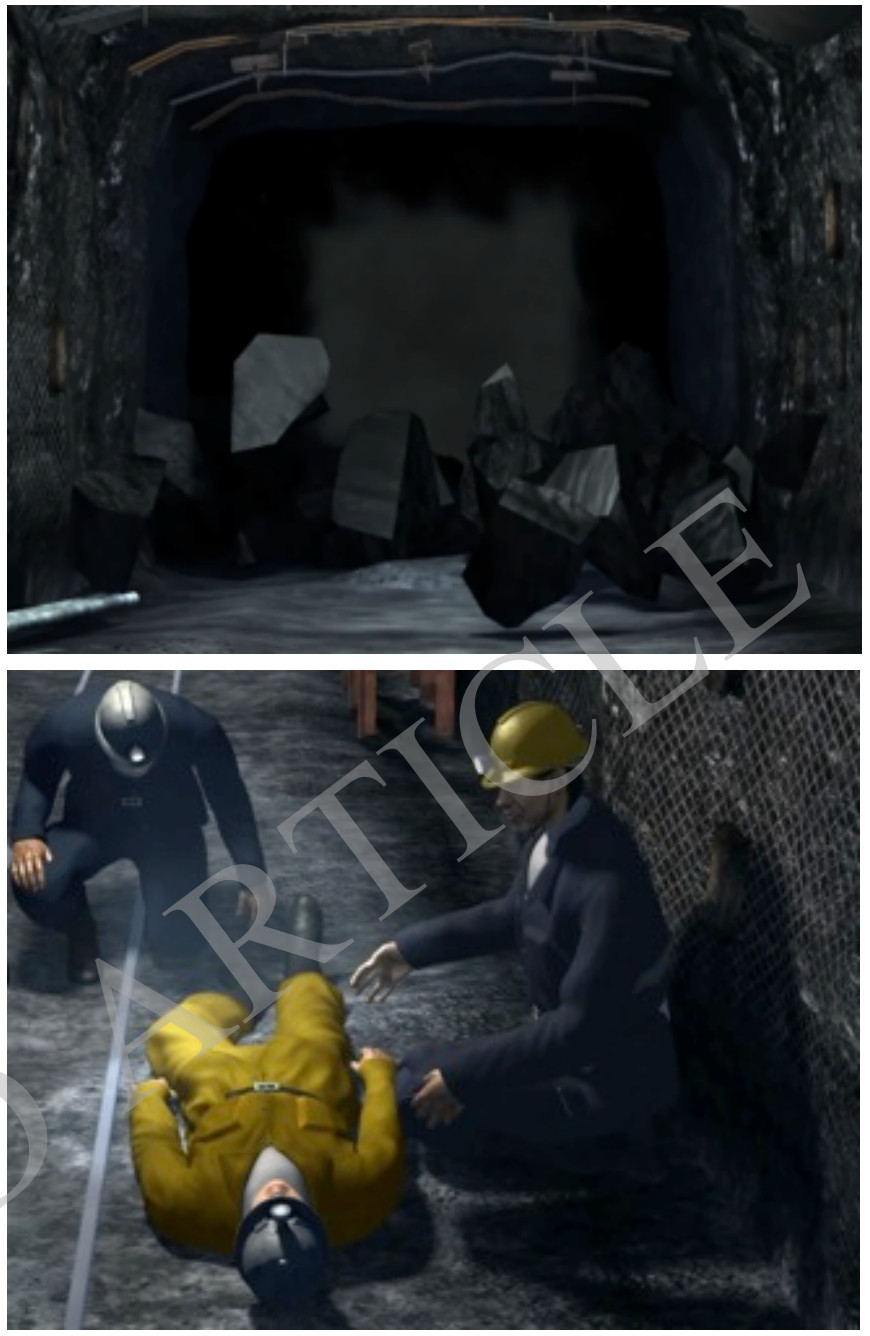

Fig. (5). The consequences arising out of the illegal operation.

\section{CURRENT \& FUTURE DEVELOPMENTS}

The $3 \mathrm{D}$ virtual simulation training system developed in this paper combining with the $3 \mathrm{D}$ stereoscopic projection technology can realize the 3D stereoscopic cinema level audio-visual effect. 3D modeling software is used to simulate the scene of the accident, play process of accident by a virtual character. Analyze the cause of the accident, and summarize preventive measures and lessons learned, strengthen the awareness of the safety of mine workers on the basis of recall and simulation of process of the accident. It promotes the safety in production and plays a good application affect.

Through the 3D virtual simulation training system for coal mines; miners can be trained in a virtual environment before they enter the underground field, accumulate underground working experiences, identify and solve problems effectively in advance and prevent disastrous accidents that are caused by human factors. At the same time, the 3D virtual simulation training system of coal mine allows users to quickly and accurately confirm the position to achieve accurate understanding of the mine environment and layout. Meanwhile, utilize three-dimensional animation 
creation capabilities to simulate rapidly the underground emergency action plans in three-dimensional environment and realize the function of planning, deploying and rehearsal of the emergency action plan when mine encounters significant security risks.

\section{CONFLICT OF INTEREST}

The authors confirm that this article content has no conflict of interest.

\section{ACKNOWLEDGEMENTS}

This research was supported by the National Key Technology R\&D Program (No.2012BAK04B09), the Fundamental Research Funds for the Central Universities (No. CDJZR13240022), the Natural Science Fund for creative research groups in China (No. 51304256; 51204216) and China Postdoctoral Science Foundation (No. 2013M540620), which are all greatly appreciated.

\section{REFERENCES}

[1] Cai, Z. The Research on Virtual Reality System of Mine Safety. Master Thesis, Hennan: Institute of Technology of Henan, 2010.

[2] Ren, N. Development and application of virtual reality on safety training system in coal mine. China Coal, 2013, 71-75.
[3] Shen, X.; Zhang, J. Visualized simulation technique in coalmine safety training. Comput. Syst. Appl., 2010, 176-179+127.

[4] Ren, C.; Zhao, Z.; Cai, D.; Wang, Q.; Xu, S. Development and application of unsafe behavior warning simulation system used in coal mine, Mining Safety Environ. Protect., 2013, 52-55.

[5] Gu, Y.; Qi, J. Design and implementation of coal mine safety simulation system based on VR technology. Coal Tech., 2012, 149151.

[6] Ren, G. Investigation and actualization of visual mine, Maser Thesis: ShanDong University of Science and Technology, 2007.

[7] Shionoya, T. Method for communication between simulators, and simulation system. W. O. Patent 2012/070143, February 13, 2014.

[8] Greifeneder, J. System and method for the configuration of a clustered simulation network. U. S. Patent 14047803, February 6, 2014.

[9] Wallace, M.W.; Zboray, D.A.; Aditjandra, A.; Webb, A.L.; Postlethwaite, D.; Lenker Z.S. Virtual reality gtaw and pipe welding simulator and setup. Google Patents, 2013.

[10] Gunter, S. Automatic context management for web applications with client side code execution. U. S. Patent 13942965, January 23, 2014

[11] Suyama, T. Robot simulator, robot teaching device and robot teaching method. W. O. Patent 2012/068448, January 23, 2014.

[12] Mee, N.A.Y. Interior simulationsystem using measuring tape tool module. W. O. Patent 2013/006455, January 23, 2014.

[13] Park, H.J. Sensing device and method used for virtual golf simulation apparatus. U.S. Patent 14004938, January 2, 2014

[14] Zhang, S. Work state virtual simulation system for electric haulage shearer based on different geological conditions. Google Patents, 2012.

[15] Hou, Y. Coal mine accident simulating method and system based on multi-intelligent agent. Google Patents, 2012. 